\title{
The Potential of Ecological Momentary Assessments in the Prediction of Suicidal Ideation: A Feasibility Study
}

\section{Other Journal Item}

\section{Author(s):}

Winkelbeiner, Stephanie; Sels, Laura; Homan, Philipp; Klee, Nina; Santhanam, Prabhakaran; Vetter, Stefan; Seifritz, Erich; Galatzer-Levy, Isaac; Kowatsch, Tobias (D); Scholz, Urte; Kleim, Birgit

Publication date:

2020-05-01

\section{Permanent link:}

https://doi.org/10.3929/ethz-b-000449552

\section{Rights / license:}

Creative Commons Attribution-NonCommercial-NoDerivatives 4.0 International

Originally published in:

Biological Psychiatry 87(S 9), https://doi.org/10.1016/j.biopsych.2020.02.1149 
The Potential of Ecological Momentary Assessments in the Prediction of Suicidal Ideation: A Feasibility Study

Stephanie Winkelbeiner ${ }^{1}$, Laura Sels ${ }^{2}$, Philipp Homan ${ }^{1}$, Nina Klee ${ }^{1}$, Prabhakaran Santhanam ${ }^{3}$, Stefan Vetter ${ }^{1}$, Erich Seifritz ${ }^{1}$, Isaac Galatzer-Levy ${ }^{4}$, Tobias Kowatsch ${ }^{3}$, Urte Scholz ${ }^{1}$, and Birgit Kleim ${ }^{1}$

${ }^{1}$ University of Zurich, ${ }^{2}$ Experimental-Clinical and Health Psychology, Faculty Psychology and Educational Sciences, Belgium, ${ }^{3}$ Center for Digital Health Interventions, ETH Zurich, ${ }^{4}$ AICure

Background: More than 800000 people commit suicide every year. This calls for better predictors and prevention. Yet, the temporal dynamics of suicidal ideation make this challenging. A solution might be ecological momentary assessments (EMA). The real-time, real-world data collection might capture the temporal dynamics and help identify predictors. We tested the feasibility of EMA in a high-risk population and examined the predictive value of sleep quality, a promising predictor and modifiable risk factor.

Methods: We included patients across diagnoses with suicidal ideations from the University Hospital of Psychiatry Zurich, Switzerland. Patients used an in-house developed app with 5-times daily EMA for 28 days during the most critical period for suicides: hospital discharge. EMA data was analyzed with a random intercept, random slope model. Results: We included 15 patients (females: $n=12$, age: $M=31.20 \pm 12.71$ years) who were clinically depressed (Beck Depression Inventory: $M=30.63 \pm 14.19$ ) and reported suicidal ideations (107 of 455

EMA in the first week, 23.5\%). Of the $455 \mathrm{EMA}$ in the first week, more than half were completed $(n=230,50.5 \%)$. We found no evidence that sleep quality predicted suicidal ideation $(\beta=-0.25$; $95 \% \mathrm{Cl},-0.59,0.04 ; \mathrm{P}=0.12)$. Conclusions: We showed that EMA allow to assess suicidal ideations, even in a high-risk population, and to capture its temporal dynamics which is important for an adequate esti-mation of suicidal ideations to identify reliable predictors and ultimately develop effective treatments. Sleep quality was not a predictor for suicidal ideation, which might be due to the limited sample size and requires further investigation in a larger sample.

Supported By: Swiss National Science Foundation Keywords: Suicidal Ideation, Sleep, Ecological Momentary Assessment 